II.

Aus dem Johanniterkrankenhause zu Neidenburg (0stpr.).

\title{
Beitrag zur operativen Behandlung des Nabelschnurbruches.
}

\author{
Von \\ Dr. Gutzeit, \\ dirig. Arzt.
}

(Mit 2 Abbildungen.)

Die operative Behandlung der Hernia funiculi umbilicalis durch Bauchschnitt, Wegnahme des Bruchsackes und Bauchdeckennaht ist nach Lindf ors ${ }^{1)}$ zuerst von 0 ken im Jahre 1810 empfohlen und 1836 von Bérard zum ersten Male mit gutem Resultat ausgeführt, aber erst seit 1881 häufiger geübt worden. Seitdem sind noch eine Anzahl anderer operativer Methoden bekannt gegeben, die größtenteils die Eröffnung des Peritoneums zu vermeiden suchen und bei der Verschiedenartigkeit des Leidens gewil ihr berechtigtes Anwendungsgebiet behalten werden.

Die Hüllen des Nabelschnurbruches werden bekanntlich von Amnion und Peritoneum gebildet, zwischen welche schleimiges Bindegewebe (Whartonsche Sulze) mit den Nabelgefäßen eingelagert ist. Seine Basis bilden die Bauchdecken, besonders die Haut in Form eines mehr oder weniger hohen, das Amnion einfassenden Ringes.

Dieser anatomische Aufbau ist desbalb wichtig, weil er allein die indes aus verschiedenen Gründen häufig ausbleibende Spontanheilung der Hernia fun. umbil. ermöglicht, indem die äußere Schicht - das Amnion - mit der Eintrocknung der Nabelschnur abstirbt und von der inneren Schicht - Peritoneum - Granulationen aufschießen, die allmählich durch Epithelisierung von der umgebenden Bauchhaut und Schrumpfung zur Bildung einer Narbe führen.

Zugleich ist die in den ersten Stunden und Tagen nach der Ge-

1) Zur Lehre vom Nabelschnurbruch und seiner Behandlung. Volkmanns Vortr. n. F. Nr. 63. 1893. 
burt eintretende physiologische Gangrän des Amnionteils des Nabelschnurbruchsacks von eminent praktischer Bedeutung für die chirurgische Therapie des Leidens, die möglichst sofort post partum einzusetzen hat, da sie später immer auf einem mehr oder weniger infizierten Operationsterrain sich abspielen muß.

Die Entscheidung, ob bei fortgeschrittener Gangrän des Bruchsacks überhaupt noch ein operativer Eingriff vorzunehmen ist, ist daher sicherlich nicht immer leicht zu treffen, zumal wenn es sich dabei noch um ein recht elendes Wesen handelt.

Es dürfte deshalb die folgende Krankengeschichte eines Kindes, bei dem die laparotomische Radikaloperation noch am 7 . Lebenstage einen recht zufriedenstellenden Erfolg hatte, nicht ohne Interesse sein.

Der anscheinend am normalen Schwangerschaftsende, mit den Zeichen der Reife am 6.5. 1903 ohne sonstige Milbbildungen geborene, sehr schwächliche Knabe von knapp 2500 gr Körpergewicht wies in der Oberbauch. gegend näher dem Proc. ensiform. als der Symphyse einen etwas ovalen Hautdefekt von $5 \mathrm{~cm}$ Breite und $6 \mathrm{~cm}$ Höhe auf. Die Bauclhhöhle war hier durch eine glasige, etwas durchscheinende, flachkuppelförmige Membran abgeschlossen, in deren unterem Teil die Nabelschnur inserierte. Die Haut endigte an der Peripherie der so gebildeten Blase mit einem regelmäßigen, allmählich sich verdünnenden, einige Millimeter hohen, scharfen Rand. Die Bauchdecken im ganzen waren ziemlich gespannt. Beim Schreien wurde die Wölbung der Membran stärker. An der Wirbelsäule nichts Auffälliges.

Der Vater des Kindes hatte 1893 sich luetiseh infiziert, war verschiedentlich behandelt und zur Zeit meiner Beobachtung symptomfrei. Die Mutter stammte aus stark tuberkulös belasteter Familie (beide Eltern starben an Phthise), hatte 1901 zwei Aborte durchgemacht, war danach gynäkologiseh behandelt und seit Anfang August 1902 zum dritten Male gravid. Im 4. und 5. Monat der Schwangerschaft hatte ich sie an mäßigen Blutungen zu behandeln, die das erste Mal 1 Tag, das zweite Mal 5 Tage anhielten und auf Opium und Bettrulie standen. Die Blutungen lagen zirka 4 Wochen auseinander. Das Kind wurde in II. Hinterhauptslage geboren. Die durch Credé entbundene Placenta zeigte makroskopisch niclits Auffälliges.

Da eine Operation bei dem sehr schwächlichen Kinde wenig Chancen bot, und die Eltern sich zunächst nicht entschließen konnten, in eine solehe zu willigen, wurde ein Schutzverband über den Nabelschnurbruelı angelegt und das Kind recht warm gebettet. Mit der Eintrocknung der Nabelschnur am 2. Lebenstage fingen die äußeren Bruchhüllen an, sich grünlichschwarz zu verfärben. Alsbald stellte sich ein höchst ïbler Geruch ein. Die Gangrän schritt rasch weiter am Bruchsack. Die bei der Weite der Bruchpforte besonders große Gefahr eines längeren Abwartens, das wohl unbedingt zur spontanen Eröffnung der Bauchhöhle geführt hätte, gab mir ein Recht, die Operation jetzt als eine eventuell lebensrettende hinzustellen und die Einwilligung der Eltern dazu zu fordern.

Am 12. 6. wurde das mittlerweile eher noch elender gewordene Kind 
in das hiesige Johanniterkrankenhaus gebracht und im gut geheizten Operationszimmer auf vorgewärmte Tücher gelegt.

Der Bruchsack wird möglichst von Sekret befreit und mit steriler Gaze bedeckt, die umgebende Haut desinfiziert, Hände, Instrumente und Operationsgebiet nach der Desinfektion mit steriler Koclisalzlösung abgespült. Der Hautring wird nun ohne Narkose in $1 / 2 \mathrm{~cm}$ Abstand von der Hautamniongrenze rundherum inzidiert, sodaß er ganz wegfällt, spritzende Gefäße sofort gefaßt und das Peritoneum eröffnet. Im unteren Teil des Schnittes treten sofort blasse, spiegelnde Dünndarmschlingen hervor, im oberen ist der Bruchsack flächenhaft mit der Leber verwachsen. Unter leichter Blutung

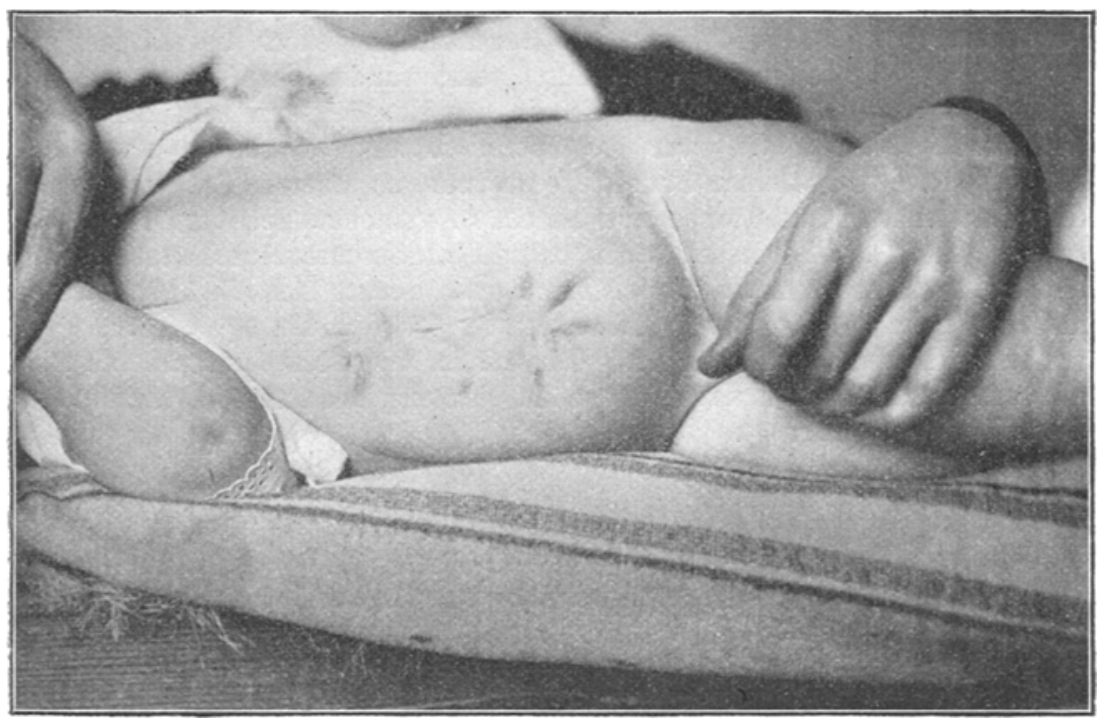

Fig. 1.

Operationsnarbe am 25. November 1903, ca. 61/2 Monat nach der Operation.

läßt er sich bis auf Lig. suspensorium und Nabelvene ablösen; letztere werden abgebunden. Die Leber. sah frisch aus; die Blutung stand auf Kompression; nur an einer Stelle wurde eine Catgutumstechung nötig. Die entstandene Laparotomiewunde klaffte nach der Entfernung des Bruchsacks außerordentlich stark; die Därme mußten mit Kochsalzkompressen zurückgehalten werden. Die Bauchdeckenränder wurden mit durch alle Schichten durchgreifenden Seidennähten breit gefaßt; das Knïpfen der Fäden gelang bei der starken Spannung nur nach mehrmaligem, kräftigstem Anziehen. Desbalb und wegen der Schwäche des Kindes wurde eine Etagennaht unterlassen. Immer wieder drängten sich bei dem mit Annäherung der Bauchdeckenränder vermehrten intraabdominalen Druck Darmschlingen in die Nahtlinie. Die Naht war so äußerst mühsam; es gelang schließlich jedoch überall die Bauchhöhle fest zu schließen. Die Wunde wird mit 
Gaze und Watte bedeckt und durch darüber gelegte Heftplasterstreifen noch möglichst entspannt.

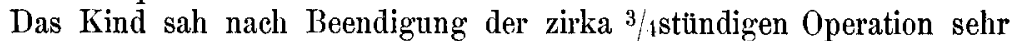
hinfällig aus, trotzdem es nicht viel Blut verloren hatte. $\mathrm{Zu}$ Hause nahm es jedoch sofort die Brust, ohne danach zu erbrechen. Am 13. 5. wurde die mütterliche Nahrung wohl infolge der vielfachen Aufregungen so gering, daß Milch gegeben werden mußte. Trotzdem befand sich das Kind verlältnismäßig wohl; der Leib blieb flach, die Darmentleerungen und Nahrunggaufnahme gut.

Am 16. 5. war übler Geruch am Verband festzustellen. Seine unteren Lagen zeigten etwas grünlichschwarzes Sekret; 2 Nähte im unteren T'eil hatten die Haut durchschnitten und ihre Ränder waren hier etwas auseinandergewichen. In der Tiefe hielten die Bauchdecken jedoch überall fest zusammen. Bei täglichem Verbandwechsel verschwanden der Geruch und die Absonderung schnell; es bildeten sich in den Hautdefekten bald reine Granulationen, und am 1. 6. war die Operationswunde vernarbt. Am 6. 6. entstand noch ein kleiner Bauchdeckenabszeß im oberen Teil der Narbe, der nach Inzision und Tamponade am 22. 6. verheilte. Ich vermutete, daß er mit den an der Leber gelassenen Catgutligaturen zusammenhänge, konnte aber Reste derselben nicht finden. Der Knabe erholte sich allmählich immer mehr, wog am 30. 7. $4200 \mathrm{gr}$, Mitte Oktober $6750 \mathrm{gr}$, am 17. 11. $7500 \mathrm{gr}$. und ist jetzt recht wohl genährt. Die $9,2 \mathrm{~cm}$ lange Narbe ist überall fest, im unteren Abschnitt strahlig und wölbt sich nirgends selbst bei kräftigstem Schreien ror (s. Fig. 1).

Die Berechtigung der Operation an sich und der angewandten Methode dürfte, obwohl durch den Erfolg erwiesen, durch eine Erörterung der bisher bekannten Verfahren zur Heilung des Nabelschnurbruches einleuchtender werden. Zugleich wird sich dabei Gelegenheit bieten, auf einige technische Einzelheiten näher einzugehen.

Fast alle Autoren, die sich mit der Hernia funiculi umbilicalis seit der antiseptischen Ära beschäftigt haben, halten die exspektative Behandlung durch Schutz- und Kompressionsverband nur bei Verweigerung der Operation, sehr breiten, nicht mehr zu vernähenden Bauchspalten oder Komplikation mit anderen tödlichen Mißbildungen für berechtigt.

Bei einem sehr großen Defekt hat Ahlfeld ${ }^{1)} 1894$ die Hautränder nach Einstülpung des ganzen Bruchsackes durch Bauchhaut und Amnion fassende Ligaturen soweit wie möglich zusammengezogen und damit Überhäutung erzielt. Dieses Verfahren macht jedenfalls auch einen Teil der größeren Spalten noch einer erfolgreichen Behandlung zugänglich.

1) Pöppelmann, Die Behandlung des Nabelschnurbruches. Inaug.-Diss. Marburg 1894. 
Bei zu ausgedehnten Entzündungserscheinungen ist natürlich auch die konservative Behandlung indiziert, vor allem bei allgemeiner Peritonitis. Jedoch wird man gut tun, solche Fälle, wenn sie nicht schon ganz verzweifelt liegen, lieber chirurgisch anzugreifen, als so sterben zu lassen, da z. B. Breus') noch bei reichlichem peritonitischen Exsudat durch seine perkutane Ligatur, Lotheißen ${ }^{2}$ ) bei einem bereits fiebernden Kinde mit peritonitischen Verklebungen durch Laparotomie und Drainage, Mikulicz ${ }^{3}$ ) bei Lebergangrän durch Resektion Heilung erzielte.

Im übrigen ist das ruhige Zuwarten bei den sonst häufig gesunden Kindern nicht zu verantworten. Die unausbleibliche Mortifikation der Amniondecke gefährdet das junge Leben durch Perforation des Sackes, Infektion des Peritoneums und der im Bruchsacke liegenden Organe, und es ist immer mehr als ein Glück zu betrachten, wenn die eingangs erwähnte und beobachtete Naturheilung tatsächlich zustande kommt. Olshausen ${ }^{4}$ ) sah sie unter 15 Fällen nur einmal sich anbahnen. Kaltenbach5) spricht sich dahin aus, daß die meisten Kinder nach Abstoßung des Nabelschnurrestes und Nekrose der dünnen Bruchsackwand an Peritonitis zugrunde gehen.

Tritt wirklich Spontanheilung ein, so erfordert sie, wie übereinstimmend berichtet wird, bei etwas größeren Brüchen eine recht lange Zeit. Die Kinder werden durch die langdauernde Wundeiterung natürlich sehr geschwächt; so starb der exspektativ behandelte Fall $01 \mathrm{~s}$ hausens (l. c.) in der 5. Woche. Ich glaube, meinem kleinen elenden Patienten wäre es ohne Operation ähnlich ergangen.

Breus (l. c) weist ferner darauf hin, daf die registrierten Spontanheilungen in der größten Mehrzahl nur als Heilungen quoad vitam, nicht aber quoad herniam anzusehen seien. Es erfolgt eben nur, wie Kroenigi ${ }^{6}$ ) es treffend ausdrïckt, eine Benarbung des Bruchsackes.

Somit besteht für die meisten Fälle von Nabelschnurbruch die

1) Die Behandlung des Nabelschnurbruches. Volkmanns Vortr.n.F. Nr. 77.1893.

2) Über Nabelsehnurbruch. Wiener klin. Rundschau. 1903. Nr. 42. S. $75 \%$.

3) Rothe, Ein nach Leberresektion geheilter Fall von gangränösem Nabelschnurbruch. Bruns Beiträge z. klin. Chirurgie. 33. Bd. 1902. S. 136.

4) Zur Therapie der Nabelschnurhemien. Arehiv f. Gynäkologie. 20. Bd. 1887. S. $443 \mathrm{ff}$.

5) Lehrbuch der Geburtshülfe. 1893.

6) Zur Behandlung des Nabelschnurbruches. Sitzungsbericht der Gesellschaft f. Geburtshülfe zu Leipzig vom 17. Oktober 1898. Zentralbl. f. Gynäkologie. Nr. 51. 1898. 
Notwendigkeit eines operativen Vorgehens. Als chirurgische Eingriffe kommen folgende in Betracht:

Die einfache zirkuläre Ligatur und die perkutane Ligatur von Breus (l. c.) können nur bei kleinen und gestielten Brüchen, d. h. kleinen Bruchpforten angewandt werden, wenn die Reposition völlig. sicher gelingt.

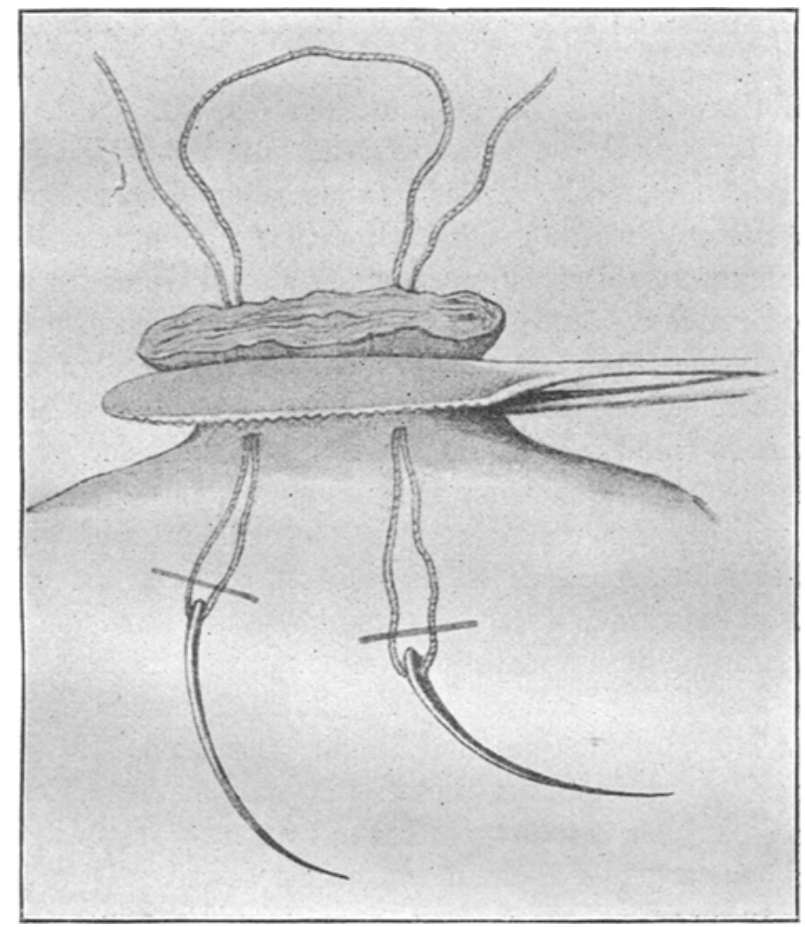

Fig. 2.

Aus Breus, Volkmanns Vortr. n. F. Nr. 77, 1893. (An den durch Striche bezeichneten Stellen werden die Fäden durchschnitten, so daß 3 Ligaturen entstehen.)

Die zirkuläre Ligatur, die jetzt wohl allseitig verlassen ist, bedarf keiner weiteren Erläuterung. Als Radikaloperation, wozu sie Pöppelmann (l. c.) und Breus (1. c.) rechnen, wird sie wohl sonst von keiner Seite angesehen, da selbst im günstigsten Falle bei hohem Hautring nur Haut und Peritoneum in die Ligatur fallen. Höchstens kommt ihr ein prophylaktischer Wert zu, indem sie bei völlig reponiblen Hernien mit dünnem Hals die Reposition sichert und den größten Teil des ohnehin absterbenden Bruchsacks beseitigt. Sie schafft da- 
durch für eine nicht gleich ausfübrbare Radikaloperation günstigere Verbältnisse und läßt es nicht zu Verwachsungen des Bruchinhalts mit der Sackwandung kommen.

Breus (1. c.) verfälırt folgendermaßen: Reposition bei uneröffnetem Bruchsack durch Ausstreichen desselben von dem erhobenen Nabelstrang aus nach der Bauchhöhle. Anlegung einer $\mathrm{K}$ öberleschen Pince hémostatique um den Hals des Bruchsacks im Bereich des Hautrings (Fig. 2). Abtragung des Bruchsacks mit der Schere. Anlegung mehrerer starker perkutaner Seidenligaturen unterhalb der Klemme. Verschorfung der Schnittfläche des Stumpfes mit dem Thermokauter; antiseptischer Verband.

Bei Anwendung des Breusschen Verfahrens ist das Abbinden von Darmteilen häufiger vorgekommen. Breus will diesen Nachteil dadurch vermeiden, daß er die Klemme nicht fester als nötig anlegt, um ihren Halt zu sichern, und den Bruchsack dann erst aufschneidet; etwa dem Bruchsack adhärierende Darmschlingen können dann noch, nach Umschneidung des anhaftenden Teils, mit dem Bruchperitoneum nach Abtrennung des Amnion versenkt werden. Er selbst hat ïbrigens in einem eigenen Falle ein Meckelsches Divertikel, dessen Natur er an den Vasa omphalomeseraica erkannte, mit abgebunden und Heilung ohne Darmfistel erzielt. Bezüglich des von Fleis chmann ${ }^{1}$ ) publizierten Falles, in welchem der Proc. vermiform. bei Anwendung der Breusschen Methode in der Ligatur eingeklemmt wurde und der Tod an Peritonitis erfolgte, meint Breus, daß sich dieses unglückliche Ereignis bei vorheriger Visitation des Bruchsacks hätte vermeiden lassen.

Doch selbst bei größter Vorsicht hat Breu s' Verfahren mehrere Nachteile. Es verbraucht mehr Gewebe als die einfache Anfrischung. Beim Fehlen eines Hautringes läßt sich die Klemme nicht anlegen, ebenso nicht bei größeren Defekten, da der Bruchsackhals wegen zu starker Spannung der Bauchdecken dann nicht mehr in eine Klemme $\mathrm{zu}$ fassen ist (vgl. Fall 2 bei $\mathrm{Br}$ eus l. c.). Mit Recht weist ferner Pöppelm an n (l. c.) darauf hin, daß die Art, wie Breus die Gefahr einer Abbindung von Teilen des Bruchinhalts vermeidet, den Vorteil seiner Methode, auch bei nicht so vollkommener Asepsis anwendbar zu sein, aufhebe; auch werde das Abbinden eines Meckelschen Divertikels nicht immer so glücklich ablaufen. Mir scheint es außerdem nicht ausgeschlossen, daß beim Anlegen der Naht durch die zusammengefaßten Bauchdeckenränder einmal ein sich in die Nahtlinie vordrängender Darmteil durchstochen wird, oder man muß die

1) Prager med. Wochenschr. 1882. Zit. n. Breus l. c. 
Nähte oberflächlich legen, dann wird aber eine feste Vernarbung nicht gewährleistet.

Am wenigsten rationell erscheint Olshausen (l. c.) die von Breus gewähite Operationsweise: "Die Anwendung einer Klammer, unterhalb welcher die Nähte durchgeführt werden, kann die Exaktheit der Nalt nur beeinträchtigen, und die Anwendung des Thermokauter, um den Bruchsack abzutragen, kann die Naht vollends illusorisch machen oder ihre Wirkung allein auf das Peritoneum beschränken." Auch K roenig (l. c.) empfiehlt das Verfahren von Breus nicht wegen der Möglichkeit von Nebenverletzungen.

Die Verschorfung mit dem Paquelin unterließ Breus übrigens in den späteren Fällen.

Tatsächlich ist die Breussche Methode kaum weiter angewandt worden, so daß Lindfors (l. c.) und Knoop l) bis 1903 nur 5 derart behandelte Fälle in ibrer Statistik zusammenstellen konnten.

Vor der einfachen zirkulären Ligatur hat sie jedenfalls den Vorzug, daß die Bedingungen zu einer festen Narbe günstiger liegen, da die Ränder der Bruchpforte gegeneinander vernäht werden. Wegen ihrer Einfachheit mag sie daher bei sicher reponiblen Brüchen mit nicht zu großer Pforte überall da, wo eine Asepsis nicht garantiert und genügende Assistenz nicht vorhanden ist, vorgenommen werden.

1887 operierte Olsha usen (l. e.) in der Absicht, die Eröffnung des Peritoneums zu vermeiden, in folgender Weise: Bei einem $31 / 2$ Stunden alten Kinde mit runder Bruchpforte von 4,5 cm Durehmesser umschnitt er diese in geringer Entfernung vom Hautrand, indem er nur die Haut durchtrennte. Der umschnittene Hautring wurde dann samt Amnion vom Peritoneum parietale abgezogen, was leicht gelang, die Whartonsche Sulze nach Möglichkeit entfernt, das Bruchsackperitoneum eingestülpt und die Bauchdecken mittels tiefgreifender und oberflächlicher Nähte zum Schluß gebracht. Die Spannung war eine recht erhebliche, so daß $1 / 3$ der Naht wieder klaffte und per granulationem heilte. Olshausen empfahl sein Verfahren nur bei reponiblen Brüchen; da das Peritoneum erhalten bleibt, soll anch für den leicht möglichen Fall einer Dehiszenz der Wunde die Heilung gesichert sein, da die vom Bauchfell aus sich bildenden Granulationen den Grund der Wunde bald erfüllen.

Wer einmal mit dem fortwährenden Vorfallen der Därme bei einer laparotomischen Radikaloperation zu kämpfen gehabt hat, wird sich der Olshausenschen Methode freuen. Leider sind in praxi ihre Erfolge keine glänzenden, ihre Ausführung häufig nicht möglich. Einmal scheitert die Trennung von Amnion und Peritoneum oft an der Nabelschnurinsertionsstelle; sobald das Amnion etwas ein-

1) Beitrag zur Therapie der Nabelschnurbrüche. Volkmanns klin. Vortr. n. F. Nr. 348. 1903 . 
getrocknet ist, ist sie überhaupt unausführbar. Kroenig (l. c.) konnte schon 12 Stunden, Runge 16 Stunden nach der Geburt Amnion und Peritoneum nicht mehr trennen. Das O Ishau sen sche Verfahren beschränkt sich daher auf die unmittelbare Zeit nach der Geburt. Ist die Gangrän des Amnion einigermalien vorgeschritten, so ist die Methode ferner nicht mehr geeignet, ,alles zu entfernen, wovon Infektion ausgehen kann" (Lindfors l. c.). Eckerlein ${ }^{2}$ ) weist bei einem von Do hrn operierten Falle darauf hin, daß in dem durch die Einfaltung. des Peritoneums entstandenen Hohlraum leicht Stagnation von Wundsekreten stattfinden könne. Dohrn erhob daher das Peritoneum zu einer Falte, die nach fortlaufender Catgutnaht abgetragen wurde. Auch bei diesem Modus entstand jedoch ein Nabelbruch von Haselnußgröße.

Wenn allerdings in den Fällen von Rettig ${ }^{3}$ ), Knauer ${ }^{4}$ ) und $\mathrm{Kroenig}$ (l. c.) trotz Irreponibilität die 01 shau sen sche Metbode versucht wurde, sich aber nicht als ausfübrbar erwies, so ist das nicht der Methode zur Last zu legen, da ihr Begründer selbst die nicht reponiblen Brüche ausdrücklich von ihr ausschließt.

1898 empfahl noch einmal Kroenig (l. c.) in der Gesellschaft für Geburtshilfe zu Leipzig warm das Verfahren Olshausens mit der Modifikation, daß Haut und Fascie angefrischt und Haut mit Haut, Fascie mit Fascie vereinigt werden. In dieser Weise ausgeführt, gab er ihr sogar den Vorzug vor der Radikaloperation durch Laparotomie. Sein Vorschlag, den ganzen Bruchsack, wenn Amnion und Peritoneum bereits verbacken wären, mit dem kurz abgeschnittenen Nabelschnurrest zu versenken und darüber Haut und Fascie zu vernähen, fand schon in der Diskussion keine Zustimmung. Sänger ${ }^{5}$ ) wies auf die erhebliche technische Schwierigkeit (bei den gestielten Hernien! Verf.) hin; auch sei dies nicht unbedenklich, da man des aseptischen $\mathrm{Zu}$ standes dieser rasch welkenden Gewebe nicht so versichert sein könne.

1903 teilte $\mathrm{Kn}$ oop (l. c.) noch eine Abänderung der Olshausenschen Operation aus der Kieler Frauenklinik mit: Der Bruchsack wurde nach Umschneidung an der Hautgrenze stumpf herauspräpariert, durch

1) Erfolgreiche Operation eines Nabelschnurbruches durch Laparotomie. Archiv f. Gynäkologie. 41. Bd. 1891. S. 121.

2) Ein Fall erfolgreich operierter Nabelschnurhernie. Zeitschr. f. Geburtshülfe u. Gynäk. 15. Bd. 1888.

3) Über angeborne Nabelschnurbrüche und ihre operative Behandlung. Inaug.-Diss. Berlin 1894.

4) Ein durch Radikaloperation geheilter Fall von Nabelschnurbruch. Geburtsh.-gyn. Gesellschaft in Wien. 1. Dez. 1896. Ref. Zentralbl.f. Gynäk. 1897. S. 54.

5) Sitzungsbericht der Gesellschaft für Geburtshïlfe zu Leipzig vom 17. Okt. 1898. Zentralbl. f. Gynäkologie. 51. Bd. 1898. 
eine Reihe versenkter Catgutnähte eingestülpt und so die Bruchpforte geschlossen, darüber die Haut mit Catgutknopfnähten vereinigt.

Trotzalledem hat sich die Olshau sen sche Methode, so sinnreich sie erdacht, sonst nicht viele Anhänger erworben.

Fast allseitig anerkannt werden dagegen die großen Vorzüge der Radikaloperation durch Laparotomie, Exstirpation des Bruchsacks und Bauchdeckennaht, wie sie Lin df o $\mathrm{rs}^{1}$ ) zuerst nachdrücklichst empfahl. „Sie ist in den meisten Fällen ausführbar, sie ändert mit einem Schlage die Situation zu der einer reinen Bauchwunde, sie gibt absolut und relativ die beste Prognose." Seine 1883 ausgesprochene Hoffnung, daß die "Radikaloperation (durch Laparotomie. Verf.) die Behandlungsmethode der Zukunft werden soll", konnte Lindf ors (l. c.) schon in einer von ihm für 1882-91 aufgestellten Statistik erfüllt sehen. Knoop (l. c.) hat diese Statistik bis 1903 fortgeführt. Danach sind insgesamt seit 188277 Fälle von behandeltem Nabelschnurbruch veröffentlicht. Es wurden behandelt:

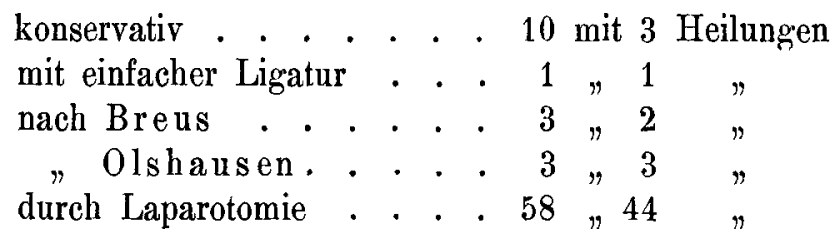

Ein ähnliches Ergebnis hat die Statistik Lotheißens (l. c.) über 91 Fälle seit der antiseptischen Zeit bis 1903. So hat also auch das letzte Jabrzehnt die Erwartungen Lin d f or s' glänzend bestätigt. Zwar bemerkt Kroenig zu Gunsten der Olshausenschen Methode, daß die Mortalität der Operation Lindf ors' sehr groß und andere weniger eingreifende Verfahren erwünscht seien. Demgegenüber muß mit Lotheißen (l. c.) betont werden, daß auf diese Art gerade die schwersten Fälle mit großer Bauchspalte operiert worden sind.

Die Technik Lindfors' war folgende: Chloroformnarkose, Reinigung des Operationsfeldes. Darauf wird der Sack erölfnet, etwaige Adhärenzen werden unterbunden und gelöst, der ganze Sack entfernt, die Hautränder angefrischt, die Nabelgefälje ligiert und schließlich die Wundränder nebst der Serosa genau und breit zusammengenäht durch tiefe und oberflächliche Suturen. 1883 führte Felsenreich ${ }^{2}$ ) eine ähnliche Radikaloperation mit bestem Erfolge aus. Interessant ist es, daß er bereits empfahl, die Hände und Instrumente nach der gründ-

1) 1. c., daselbst sind auch die früheren Arbeiten Lindfors' über dieses Thema zu ersehen.

2) Zur operativen Behandlung der Hernia funiculi umbilicalis. Wiener med. Presse. 1883. Nr. 17. S. $532 \mathrm{ff}$. 
lichen Desinfektion in abgekochtem Wasser abzuspülen, um den kindlichen Organismus nicht durch Karbolsäure zu schädigen.

Runge (l. e.) umschnitt den Bruch im Hautrande $1 / 2 \mathrm{~cm}$ vom Amnionansatz, exzidierte auf diese Weise den Bruchsack, reponierte den Inhalt und vernähte die Bauchwunde. Es ist dieses Verfahren bei nicht mehr ganz unverdächtigen Bruchbüllen in Rücksicht auf die Asepsis der Bruchsackspaltung unbedingt vorzuziehen. Man macht sich so auch die Bruchpforte zugänglich, ohne den Bruchsack außen anrühren zu dürfen, und kann durch ihre Erweiterung an einer günstig. gelegenen Stelle eine etwaige Einklemmung lösen. Diese Schnittführung wurde z. B. bei 3 inkarzerierten Nabelschnurhernien in der chirurgischen Abteilung des städtischen Krankenhauses am Urban zu Berlin mit Erfolg angewandt, wie Weyprecht ${ }^{1}$ ) berichtet.

Solange der Bruchsack noch nicht völlig umschnitten und entfernt ist, sind die Baucheingeweide leicht zurückzuhalten. Nach Wegfall des Bruchsacks und bei großen Spalten macht dies, wie auch in meinem Falle, Schwierigkeit. Ich möchte daher bei irgendwie zu befürchtender starker Spannung, bei Operationen ohne Narkose raten, die Umschneidung erst an kleinen gegenüberliegenden Stellen auszuführen und die bei Laparotomien üblichen Haltenähte durch die Bauchdeckenränder samt Peritoneum zu legen. Man hat an ihnen nach Wegfall des Bruchsacks eine gute Handhabe, um den Eingeweidevorfall zu verhüten bezw. zu beschränken.

Eine schichtweise Bauchdeckennaht wird sich bei starker Spannung nicht ermöglichen lassen. Gewiß wird man, wie Kroenig ${ }^{2}$, $\mathrm{Zw}$ ei$\mathrm{fel}^{2}$ ) und $\mathrm{S} \ddot{a} \mathrm{nger}{ }^{2}$ ) dies verlangen, wenn möglich, Peritoneum und Fascie isoliert nähen. Unbedingt erforderlich ist es nicht und verlängert immerhin den an und für sich schweren Eingriff nicht unbedeutend. Durch den Erfolg in praxi ist jedenfalls nachgewiesen, daß Haut, Fascie und Bauchfell zusammenfassende Nähte eine völlig feste Narbe geben; auch bei meinem kleinen Patienten war dies der Fall. Selbstverständlich muß dabei immer der ganze Hautring exzidiert sein, damit auch die Fascie angefrischt wird. Krukenberg ${ }^{3}$ ) beobachtete bei einem außergewöhnlich hohen Hautring von $2 \mathrm{~cm}$, weil er nur Haut und Peritoneum in die Naht falte, naturgemäl eine kuppelförmige Hervorwölbung der Narbe.

1) Erfahrungen über die Operation des eingeklemmten Bruches. Langenbecks Archiv. 71. Bd. Heft 1. 1903.

2) Gesellschaft für Geburtshülfe. Sitzung vom 17. Oktober 1898. Zentralbl. f. Gynäk. Nr. 51. 1898.

3) Zur Therapie der Hernia funic. umbil. Archiv f. Gynäk. 20. Bd. 1882. S.269. 
Auch aus Gründen der Asepsis wird man den Schnitt nicht zu nahe an die Hautamniongrenze legen.

Eine eigenartige Bauchdeckennaht wandte Lotheißen (1. c.) in seinem Falle an, indem er Peritoneum und Fascie mit einer, die Haut durch eine zweite Tabaksbeutelnaht zusammenzog; allerdings handelte es sich um eine kleine Bruchpforte. Die Tabaksbeutelnaht zum Verschluß des Peritoneums nach Laparotonie hatte schon de Quervain ${ }^{1)}$ empfohlen in der Absicht, dadurch die Peritonealwunde auf ein Minimum zu verkleinern und die Gefahr der Verwachsungen von Netz und Darm an der Innenseite der Bauchwand zu vermindern. Bei größeren Bauchspalten ist indes das Verfahren selbst auf das Peritoneum allein deshalb nicht anzuwanden, weil dasselbe in der Nabelgegend und an der Linea alba kaum verschieblich ist ${ }^{2}$ ).

Bei Verwachsung von Bruchinhalt und Sack wird man je nach den gegebenen Verhältnissen chirurgisch verfahren. So resezierte $\mathrm{Mikulicz}{ }^{3}$ ) ein irreponibles gangränverdächtigtes Leberstïck. Kü st$\left.n e{ }^{4}\right)$ umschnitt ein dem Dünndarm adhärentes Brucksacksegment, trennte von letzterem die Amnionbekleidung, löste eine Leberadhäsion scharf mit der Schere, mußte aber den prolabierten Leberteil nachträglich resezieren, da er wegen seiner Gestalt trotz erweiterter Bruchpforte in der Bauchhöhle nur unter starker Biegung unterzubringen gewesen wäre.

$\mathrm{Kroenig}$ (l. c.) versenkte 12 Stunden post partum unbeschadet einen der Leber adhärierenden Bruchsackteil, ohne das Amnion zu entfernen; doch dürfte sich dies Verfahren, wie schon Sänger in der Diskussion bemerkte, wenn überhaupt, nur für ganz frische, sicher aseptische Fälle eignen.

Ein mit Lumen ausgestattetes $\mathrm{Meck}$ elsches Divertikel ist zentralwärts einzustälpen und zu übernähen.

Jedenfalls wird bei allen solchen Verwachsungen nur die intraperitoneale Radikaloperation die nötige Übersicht geben und richtiges chirurgisches Vorgehen ermöglichen.

Bei Inkarzerationen oder da, wo die Enge des Nabelrings die Reposition verhindert, ist selbstverständlich keine andere Methode anwendbar; sie kommt allein bei größeren, noch zu vereinigenden Bauchspalten in Betracht.

Schon aus diesen Ausführungen geht hervor, daß die intraperitoneale Radikaloperation die Methode der Wahl ist, da sie bei allen Arten

1) ZurVerwendung der Tabaksbeutelnabt. Zentralbl. f. Chir. Nr. 27. 1899. S. 753 .

2) Jöss el-Waldeyer, Topograph. Anatomie. II. 1899. S. 211.

3) Rothe, Ein nach Leberrescktion geheilter Fall von Nabelschnurbruch v. Bruns Beiträge z. klin. Chírurgie. 33. Bd. 1902. S. 136.

4) Operation ciner Nabclschnurhernie init Resektion des vorgefallenen Leberlappens. Zentralbl. f. Gynäk. Nr. 1. 1901. 
von Nabelschnurbrüchen aufer den überbaupt nicht mehr oder nur nach Ahlfelds Verfahren operablen Fällen anwendbar ist.

Sie schafft die einfachsten Wundverhältnisse und gibt - technisch richtige Ausführung vorausgesetzt - feste Narben.

Die Heilungsdauer wird 3-4 Wochen nicht überschreiten, wenn keine Komplikationen eintreten.

Ich war ferner recht freudig erstaunt, wie bald nach der Operation sich mein kleiner, elender Patient erholte, trotzdem der Eingriff ihn sichtlich erschöpft hatte. Auch $\mathrm{Runge}$ fand den Verlauf bei seinem gut entwickelten und lebensfrischen Kinde "wider Erwarten" günstig. Der Grund dafür scheint mir hauptsächlich darin zu liegen, daß die Entfernung eines zumal in der Nähe der Nabelgefäße gangräneszierenden Gewebsstickes und die Lagerung der mit dem Bruchsack z. T. verklebten, aber schlecht geschützten, mehr durch ihn gezerrten und beengten Eingeweide hinter gesunder Bauchwand für die Kinder einen ganz wesentlichen Vorteil bedeutet.

Im Prinzip ohne Narkose zu operieren, wie es $Z$ we if el (l. c.) verlangt, ist gewiß berechtigt. Doch wird bei kräftigen Kindern und großer Bruchpforte - demgemäß Spannung - die Ausführung der Operation durch die Narkose wesentlich erleichtert werden. Die Kinder vertragen sie zumeist gut. In meinem Falle wäre ich schwerlich ohne sie zum Ziel gelangt, wenn das Kind nicht zuletzt so schwach geworden wäre, daß von Muskelspannungen nicht mehr viel zu merken war. Zum Schluß möchte ich noch kurz die Frage der Ätiologie des Nabelschnurbruches in Rïcksicht auf meinen Fall erörtern.

Von den Theorien, die auf entwicklungsgeschichtliche Vorgänge zurückgreifen, hatte die von $\mathrm{A}$ hlfeld die allgemeinste Anerkennung gefunden. Sie stützte sich der Hauptsache nach auf das physiologische Austreten und Verweilen des Darmes in der Nabelschnurscheide in den ersten Wochen des embryonalen Lebens. Im "Nabelschnurbruch" sollte dieser fötale Zustand, der sich sonst etwa in der 10. Woche zurückbildet, über die Geburt hinaus erhalten sein und zwar infolge einer abnorm langen Persistenz des Ductus omphalomesentericus, die das Zurücktreten des Darmes in die Bauchhöhle und die Verkleinerung des Nabelringes verhindere.

Schon Buschan') führte gegen diese Theorie an, daß Darmdivertikel oder Überreste eines solchen bei Nabelschnurbrüchen selten gefunden werden, der Nabelstrang auch meist seitlich dem Bruchsacke ansitze, sodaß eine Zugwirkung des in ihm verlaufenden Dotterstranges schwer anzunehmen sei.

1) Über Hernia funiculi umbilicalis. Dissert. Breslau 1887. 
Als wichtigste Tatsache gegen A hl f eld s Theorie fügte As ch of f ${ }^{1}$ ) 1896 hinzu, daß die Leber fast ebenso oft wie der Darm als Bruchinhalt $\mathrm{zu}$ finden ist. Auf Grund seiner anatomischen Untersuchungen und entwicklungsgeschichtlichen Studien kam er zu dem Resultat, daß die Leber infolge von Störungen der Bauchwandanlage im Bruchsacke angelegt und nicht in ihn vorgefallen sei. Die Verbindung der Leber mit dem Bruchsacke ist nach ihm nicht auf sogenannte fötale Peritonitis, sondern auf zu geringe Abspaltung zurückzuführen 2). A sch off sagt weiter: „Es kann sich in allen Fällen von sogenanntem Nabelschnurbruch nicht um eine abnorme Weite der Nabelschnurscheide im engeren Sinne handeln, sondern der Bruchsack wird von der ganzen vorderen Banchwand gebildet. Nur die Kleinheit derselben, die Bekleidung mit einer Decke, wie sie die Hülle der Nabelschnur bildet, täuscht die Annahme vor, daß die Nabelschnurscheide trichterförmig erweitert wäre. Wenn wir aber bedenken, daß bei den gewöhnlichen Formen von Nabelschnurbruch - ich sehe von den ganz kleinen Ausstülpungen der Nabelgegend ab - das Gebiet zwischen dem Processus ensiformis und dem Nabelgefäßansatz, der sich überwiegend an der unteren Ringhälfte der Bruchpforte befindet, das erweiterte ist, so müssen wir jeden Anteil der eigentlichen Nabelschnur zurïckweisen und erklären, die obere Hälfte der Bauchwand, zwischen Brustgrenze und Nabel gelegen, ist der Sitz des Bruches. Es handelt sich aber, wie bekannt, nicht um die herniöse Vorstülpung einer Peritonealtasche mit Eingeweideinhalt, denn die Bauchwände gehen allmählich in den Bruchsack über, auch nicht um mangelhafte Anlage der Abdominalmuskulatur, da sie oft nachgewiesen ist, sondern um eine zu weite Anlage der Bauchwand."

Auch in meinem Falle machte der flachkuppelförmige größtenteils oberhalb des Nabels gelegene "Bruchsack", dem die Nabelschnur unten gewissermaßen als Appendix ansaß, nicht den Eindruck einer erweiterten Nabelschnurscheide. Dotterstrangreste fehlten; nur die Leber war mit dem Bruchsacke verbunden, die Därme völlig frei; peritonitische Erscheinungen, die die Leberverwachsung erklärt hätten, waren nicht vorhanden.

Berücksichtigen wir dieses, so findet der von mir operierte „Nabelschnurbruch" in den Forschungsergebnissen von Asch off jedenfalls eine bessere entwicklungsgeschichtliche Erklärung, als durch die Ansicht Ahlfelds, für welche er sichere Stützpunkte nicht bietet.

1) Über das Verhältnis der Leber und des Zwerchfells zu den Nabelschnurund Bauchbrïchen. Virchows Archiv. 144. Bd. 1896. S. $511 \mathrm{ff}$.

2) Die ausführliche Begrïndung muß im Original nachgelesen werden. 